Article

\title{
Rapid Determination of Optimal Conditions in a Continuous Flow Reactor Using Process Analytical Technology
}

\author{
Michael F. Roberto ${ }^{1}$, Thomas I. Dearing ${ }^{2}$, Charles W. Branham ${ }^{2}$, Olav Bleie ${ }^{3,4}$ \\ and Brian J. Marquardt ${ }^{2, *}$
}

1 Department of Chemistry, University of Washington, 4000 15th Ave NE, Seattle, WA 98105, USA; E-Mail: mroberto@uw.edu

2 Applied Physics Laboratory, University of Washington, 1013 NE 40th Street, Seattle, WA 98105, USA; E-Mails: tdearing@ apl.uw.edu (T.I.D.); cbranham@apl.uw.edu (C.W.B.)

3 Tel-Tek, Kjølnes Ring 30, Porsgrunn 3918, Norway; E-Mail: olav.bleie@gmail.com

4 Department of Chemistry, University of Bergen, P.O. Box 7800, Bergen NO-5020, Norway

* Author to whom correspondence should be addressed; E-Mail: marquardt@ apl.uw.edu; Tel.: +1-206-685-0112; Fax: +1-206-616-3463.

Received: 5 November 2013; in revised form: 13 December 2013 / Accepted: 16 December 2013 / Published: 27 December 2013

\begin{abstract}
Continuous flow reactors (CFRs) are an emerging technology that offer several advantages over traditional batch synthesis methods, including more efficient mixing schemes, rapid heat transfer, and increased user safety. Of particular interest to the specialty chemical and pharmaceutical manufacturing industries is the significantly improved reliability and product reproducibility over time. CFR reproducibility can be attributed to the reactors achieving and maintaining a steady state once all physical and chemical conditions have stabilized. This work describes the implementation of a smart CFR with univariate physical and multivariate chemical monitoring that allows for rapid determination of steady state, requiring less than one minute. Additionally, the use of process analytical technology further enabled a significant reduction in the time and cost associated with offline validation methods. The technology implemented for this study is chemistry and hardware agnostic, making this approach a viable means of optimizing the conditions of any CFR.
\end{abstract}

Keywords: continuous flow; Raman spectroscopy; process optimization 


\section{Introduction}

Currently, the majority of pharmaceutical and specialty chemical manufacturing is performed in large volume batch reactors. These reactors are limited by a number of challenges: Poor mixing, large temperature gradients, potentially inaccurate serial additions, safety and pressure hazards, and resource-intensive validation procedures requiring man-hours, time, and manual sampling for quality assurance and control. Many of these challenges are mitigated using continuous flow reactors (CFRs). CFRs are small volume flow cells optimized for continuous, consistent production of a target compound. The volumes and channels of CFRs can range from milliliter to microliter scale [1]. Milliliter-scale CFRs typically employ reaction plates designed for turbulent mixing (Figure 1a). Reaction plates are typically manufactured with millimeter-wide channels and a high surface-to-volume interface for a multi-fold reduction in the average distance to temperature control channels (Figure 1b). The design results in a reduction of temperature gradients and improved homogeneity in CFRs [2-4]. It has also been shown that parallel addition of reagents removes variation introduced by operators, and a closed system is less affected by environmental variation [5]. Roberto et al. have previously demonstrated a $75 \%$ reduction in reaction time in continuous flow when compared to a batch reactor [6].

Figure 1. (a) Side view of continuous flow reactor mixing chip showing heat exchange and mixing areas. Each area has a width of $1.5 \mathrm{~mm}$, and are separated by $1 \mathrm{~mm}$ of glass; (b) Top view of continuous flow reactor mixing chip. Heart-shaped mixers are employed to ensure turbulent mixing throughout.

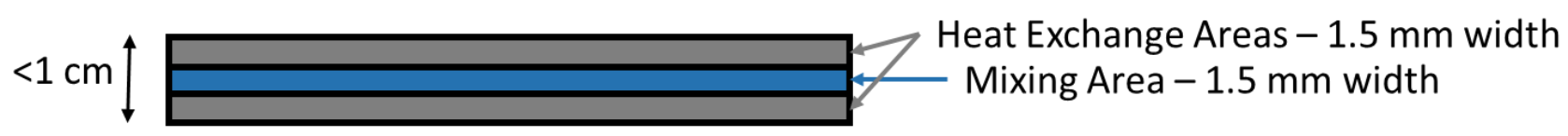

(a)

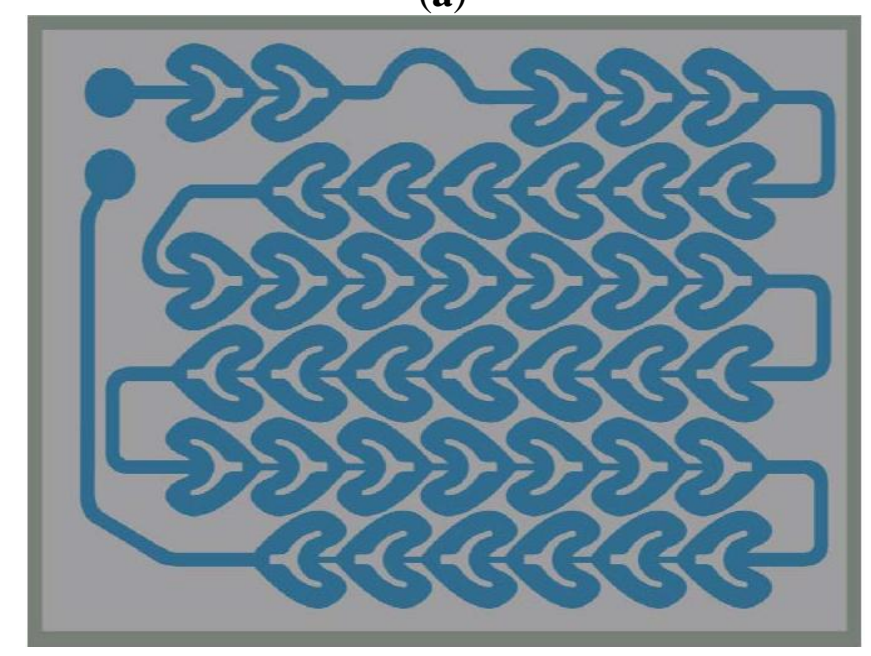

(b)

All of these advantages assume that a reactor system is in steady state. A steady state continuous flow system is one that is physically stable with regards to the variables of pressure, flow rate, mixing, and temperature. This results in controlled chemical production with reproducible results. Such a system offers significant advantages over batch reactors that fluctuate between different production 
runs. Currently, CFRs are determined to be at steady state by either a univariate method detecting physical stability in the reactor or a multivariate method determining the stability of chemical output [1,5,7-9]. Chemical measurements frequently require aliquots for offline validation to determine steady state. Uncertainty in reactor steady state requires multiple measurements, further extending analysis time. This can be especially problematic when attempting to determine optimal reactor conditions that require the exploration of a large design space. Physical measurements, while rapid, cannot effectively detect problems associated with product quality. Reactor systems are not currently constructed with interfaces to accommodate process analytical technology (PAT). Instead, current physical stability detection methods largely rely on feedback from the temperature or flow controllers without dedicated analytics.

A continuous flow system was designed that expands traditional physical monitoring while implementing real-time chemical monitoring to accurately and rapidly determine steady state. Monitoring of any process requires a reproducible sampling interface for reliable results. New Sampling/Sensor Initiative (NeSSI) sampling systems have previously been shown to be effective tools for enabling process monitoring in fluid and gas systems [6,10], and as interfaces for continuous flow reactor analytics [6]. NeSSI is a modular platform allowing for simple expansion or rearrangement of a sampling platform. This enables rapid prototyping and development of continuous flow reactor systems. The coupling of NeSSI with appropriate sensors creates a dependable interface for the sampling of a flow channel [10,11].

Chromatographic methods are the most frequently used validation methods to determine chemical steady state and conversion in CFRs [5,7-9,12,13]. These methods yield reproducible results but can have analysis times in excess of fifteen minutes after sample collection. Confirmation of chemical steady state in a CFR over multiple aliquots requires an hour or more using chromatographic methods. A design of experiments (DoE) with 25 points would require more than a day to determine optimal conditions. Raman spectroscopy has previously been applied and proven effective for in-line process monitoring of flow systems [9,14-16]. Recent work by Roberto et al. has shown that Raman spectroscopy is a viable method for monitoring chemical yield in a CFR [6]. Deconvolution of Raman spectral data is challenging in complex chemical systems. Chemometric tools such as principal component analysis (PCA) are often used to process and analyze this spectral data quickly and effectively [17,18]. Used in tandem, Raman spectroscopy and PCA allow for robust process monitoring and effective process control [18-21].

The focus of this investigation was to demonstrate that the combination of NeSSI with PAT and multivariate chemical monitoring offers significant advantages for optimizing chemical design space when compared with systems that employ physical or chemical techniques alone. Additionally, methods developed show potential for quality control and upset detection in production-scale facilities. Critical to the success of this project was the construction and design of a smart reactor system for monitoring and control of a defined chemistry with PAT. To demonstrate the smart reactor system, the Moffatt-Swern oxidation of 1-phenylethanol (Figure 2) was investigated. The formation of the highly unstable intermediates $\mathbf{3}$ and $\mathbf{5}$ is highly exothermic, requiring accurate temperature control [12,13]. The overall results demonstrate that the application of PAT to accurately detect physical and chemical stability in CFRs improved the time required to determine steady state by more than an order of magnitude. This reduced the time required to process the DoE and determine optimal reactor conditions. 
The final goal of this project was to develop a procedure for optimizing CFRs independent of the chemistry performed.

Figure 2. The Swern oxidation. The center column (green background) shows the desired chemical path, with added reagents shown in black boxes. The outer columns (red background) show the potential chemical pathways for side-product formation ( 8 and $\mathbf{9})$.

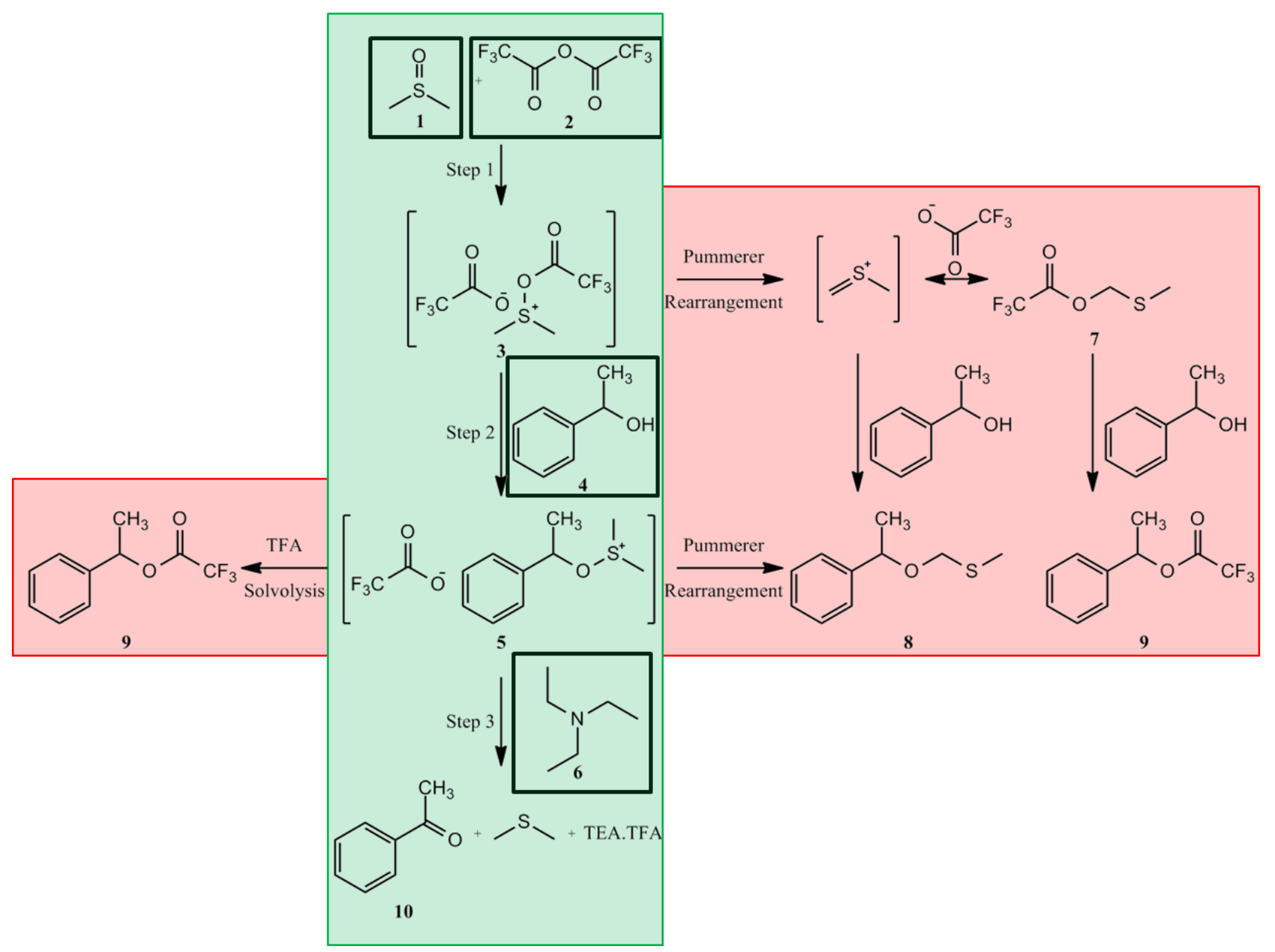

\section{Experimental Section}

The continuous flow reactor (CFR) was comprised of 4 main components: The Intraflow NeSSI sampling system, the analytics and controllers, the chemical mixers, and the control software. The Intraflow sampling system, provided by Parker Hannifin (Cleveland, OH, USA), consisted of four identical reagent lines and a product line. Four reagents: $1.0 \mathrm{M}$ trifluoroacetate anhydride (Sigma-Aldrich, St. Louis, MO, USA), 1.1 M DMSO (Sigma-Aldrich, St. Louis, MO, USA), 1.0 M S-1-phenylethanol (Sigma-Aldrich, St. Louis, MO, USA), and 2.4 M triethylamine (Sigma-Aldrich, St. Louis, MO, USA) all in dichloromethane (Sigma-Aldrich, St. Louis, MO, USA) are pumped into the system by HPLC Pumps (two FLOM FS10A HPLC pumps, FLOM USA, San Diego, CA, USA, and two SSI Series I HPLC pumps, SSI, State College, PA, USA). These pumps actively controlled the residence time and stoichiometry of the reactor system. Pressure relief valves were installed on each line to ensure that the reactor did not overpressurize. Temperature was controlled by a Huber Tango heat-exchanger (Huber USA, Northport, NY, USA) and was pumped across three sequential Corning 
LF Reactor plates (Corning Glass, Avon, France), each with a reactor volume of $1 \mathrm{~mL}$, that included 38 heart-shaped mixers per plate (Figure $1 \mathrm{~b}$ ) to ensure turbulent mixing throughout.

The analytics on each of the four reagent line were as follows: A pressure transducer (HEISE, Stratford, CT, USA), a flow meter (FCI Incorporated, San Marcos, CA, USA), and a 0.5" O.D. Raman ball-probe (Marqmetrix, Seattle, WA, USA) were interfaced to NeSSI via a fiber optic cable. The heat exchange lines were equipped with a thermocouple (Omega Engineering, Stamford, CT, USA) before and after the reactor plates to determine the temperature loss across the reactor. The product line NeSSI system contained a thermocouple and a 0.5" O.D. Raman ball-probe. A four-channel $785 \mathrm{~nm}$ Raman system, supplied by Kaiser Optical Systems (Ann Arbor, MI, USA) was used to collect Raman spectra from the four ball-probes mounted on the reactor sequentially. The sensors and controllers, with the exception of the Raman instrument, were all monitored and controlled via an in-house designed LabVIEW 7.5 (National Instruments Corporation, Austin, TX, USA) GUI environment. All of the analytics were interfaced to the reaction flow via NeSSI sampling system. The sensors and digital controllers were queried once per second; the GUI reported temperature, pressure, and flow information in real-time.

Raman investigation of the reaction chemistry was performed prior to exploring the full temperature and flow rate design space. Spectra were collected by performing chemistry at a constant flow rate of $4 \mathrm{~mL} / \mathrm{min}$ from $-20{ }^{\circ} \mathrm{C}$ to $20{ }^{\circ} \mathrm{C}$ at $10{ }^{\circ} \mathrm{C}$ intervals. Five Raman spectra were recorded; each consisting of five one-second exposures, for a total of 25 calibration spectra. All calibration samples were validated on an Agilent 1100 Series HPLC (Agilent Technologies, Santa Clara, CA, USA) using a reverse-phase column (Agilent C18 Column, Santa Clara, CA, USA). The liquid chromatography was performed using two mobile phases: $0.1 \%$ acetic acid in water and acetonitrile. A constant flow of $5 \%$ acetonitrile for $2 \mathrm{~min}$, then a gradient of 5\% acetonitrile to $95 \%$ acetonitrile over $5.5 \mathrm{~min}$, followed by $2 \mathrm{~min}$ constant flow resulted in clearly defined peaks for $S$-1-phenylethanol (4), acetophenone (10), and side-products $\mathbf{8}$ and $\mathbf{9}$. Acetophenone peak area was used to determine reaction progress.

Raman spectra were imported into MATLAB 7.5 (Mathworks, Natick, MA, USA) for analysis and modeling. All spectra were preprocessed using a 1st order baseline removal to remove variations between spectra. PCA was performed with PLS Toolbox 5.0 (Eigenvector Research, Inc., Wenatchee, WA, USA) using mean-centered, background corrected spectra to monitor reactor steady state via changes in the multivariate scores. After establishing a system for accurately detecting chemical steady state, the full 25 point design space of flow rate $(2-10 \mathrm{~mL} / \mathrm{min}$ at $2 \mathrm{~mL} / \mathrm{min}$ increments) and temperature $\left(-20{ }^{\circ} \mathrm{C}\right.$ to $20{ }^{\circ} \mathrm{C}$ at $10{ }^{\circ} \mathrm{C}$ intervals $)$ was explored via the control software.

\section{Results and Discussion}

A system was developed using a CFR, NeSSI sampling system, and PAT to monitor chemical and physical variation in real-time (Figure 3). The PAT served two purposes: To determine reaction steady state, and to detect process upsets in the reactor. The variables temperature, pressure, and flow were recorded at one second intervals at specific locations in the reactor (Figure 3). When the lowest deviation of each physical measurement had been obtained the reactor was considered physically stable. 
Figure 3. Schematic of continuous flow reactor system. Each of the four reagent lines have the same analytics present: Flow meter, pressure sensor, Raman probe, and valves for operator safety. The first two reagent lines combine in the first reactor, with each subsequent line adding a reagent to the next reactor. The product line contains a Raman probe, a pressure regulator, and a pneumatic valve that is able to send the chemical output to either product collection or waste.

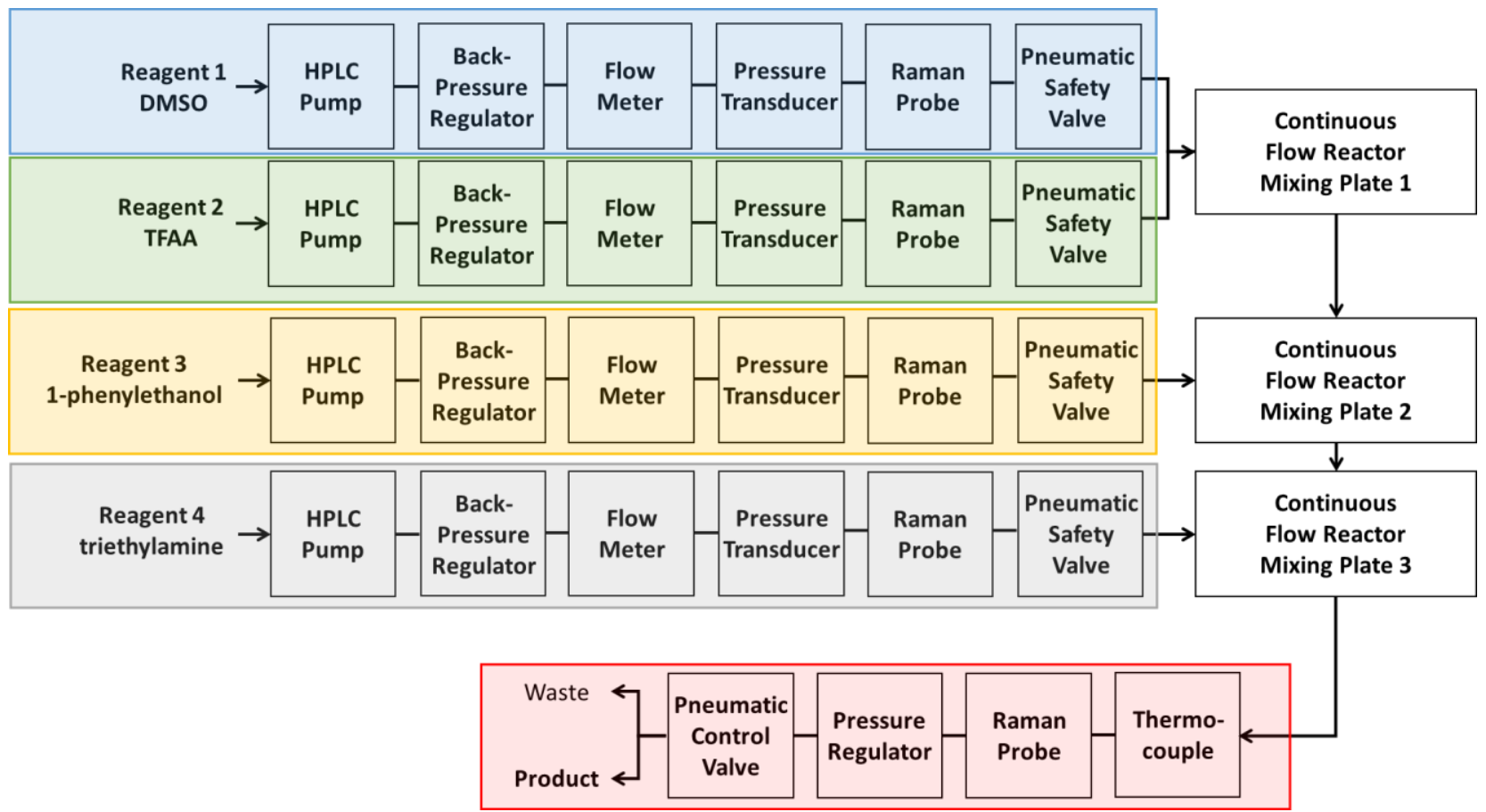

Upon reaching physical stability the reactor requires a lead time in order for the physical changes to be reflected in the chemical output downstream. Lead time is dependent upon the flow rate and the total volume of the reactor system by the equation:

$$
\text { Time } \boldsymbol{\alpha} \frac{\text { Reactor Volume }}{\text { Flow Rate }}
$$

A Raman ball probe on the product line (Figure 3) performed spectral acquisitions every $15 \mathrm{~s}$. Raman spectroscopy is particularly suitable for monitoring the Swern oxidation due to the strength of the $\mathrm{C}=\mathrm{O}$ band at $1691 \mathrm{~cm}^{-1}$ (Figure 4). Upon exiting the final reactor plate, all chemicals rapidly reached room temperature in the NeSSI sampling system prior to Raman analysis. This was confirmed by a thermocouple upstream of the Raman ball-probe on the product line. Chemical stability was determined via monitoring of the first PCA score from the Raman spectra in real-time. Four sequential scores within an acceptable statistical confidence established chemical steady state (approximately $1 \mathrm{~min}$ of sample time). Upon attaining chemical steady state, a single HPLC sample was extracted and analyzed to quantitatively determine the yield of product. 
Figure 4. Wavelength subset of Raman spectra at five different temperatures. While the entire spectrum was used for PCA modeling, this region in particular shows the temperature dependence of the production of acetophenone, with peaks at $1691 \mathrm{~cm}^{-1}$ and $1602 \mathrm{~cm}^{-1}$.

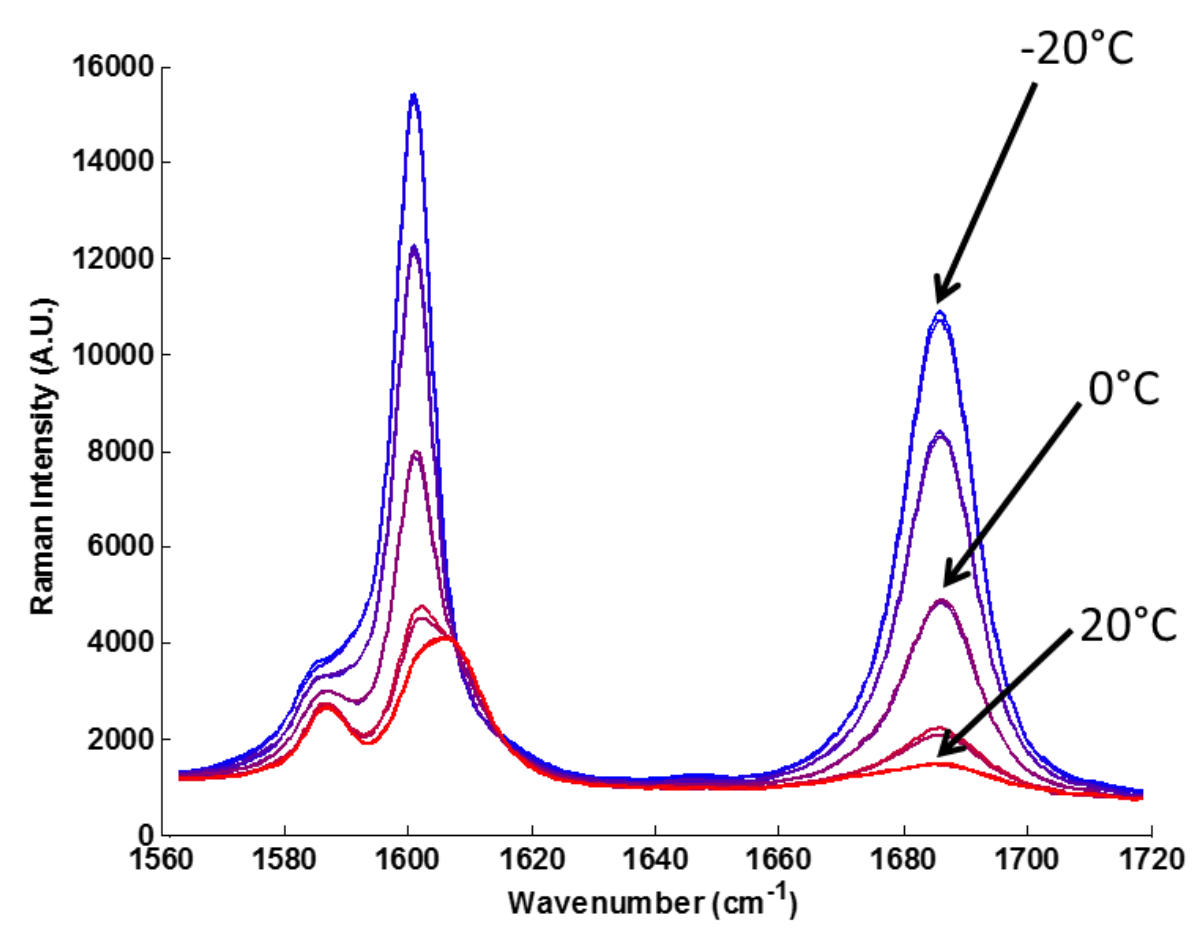

An example of how physical stability and chemical steady state relate to each other is shown in Figure 5. The dark dotted line shows the measured pressure in one of the four reagent lines. At $3 \mathrm{~min}$, the flow rates of the four reagents were doubled from 2 to $4 \mathrm{~mL} / \mathrm{min}$. Physical stability is established in less than one minute. The first change in the PCA scores is observed at 4 min, shown in the lighter dotted line. Chemical steady state is reached by $13 \mathrm{~min}, 10 \mathrm{~min}$ after the change in physical conditions. Determining chemical steady state using HPLC would have significantly increased the time to complete reactor optimization. Four consecutive HPLC measurements require an analysis cycle of one hour. This is 60 times longer than Raman monitoring to determine steady state. The real-time Raman information enabled a more effective use of HPLC for validation. Using this technique to rapidly and accurately determine steady state, a 25-point design space was explored in under four hours.

The chemical yield results obtained by using offline HPLC are shown in Table 1. In batch, at temperatures above $-30{ }^{\circ} \mathrm{C}$, no significant product yield was detected $(<2 \%)$ [13]. Within the CFR at temperatures above $-30{ }^{\circ} \mathrm{C}$ the Moffat Swern oxidation had yields ranging from $81 \%$ at $-20{ }^{\circ} \mathrm{C}$ to $37 \%$ at $0{ }^{\circ} \mathrm{C}$. As expected due to the exothermic nature of the reaction, yield decreases with increasing temperature. The unstable nature of intermediates $\mathbf{3}$ and $\mathbf{5}$ also resulted in lower chemical yield at slower flow rates (Table 1). This effect has been previously reported by Yoshida et al. [12] and Kemperman et al. [13]. 
Figure 5. Pressure response in a reagent line (dark dotted line) and chemical yield information (light dotted line) that demonstrate physical and chemical steady states and changes in the system. At $3 \mathrm{~min}$, the flow rates of the four reagent lines were doubled. The pressure sensor indicates physical steady state occurring in the reactor at $4 \mathrm{~min}$, and the chemical yield response demonstrates chemical steady state occurring in the reactor at $13 \mathrm{~min}$.

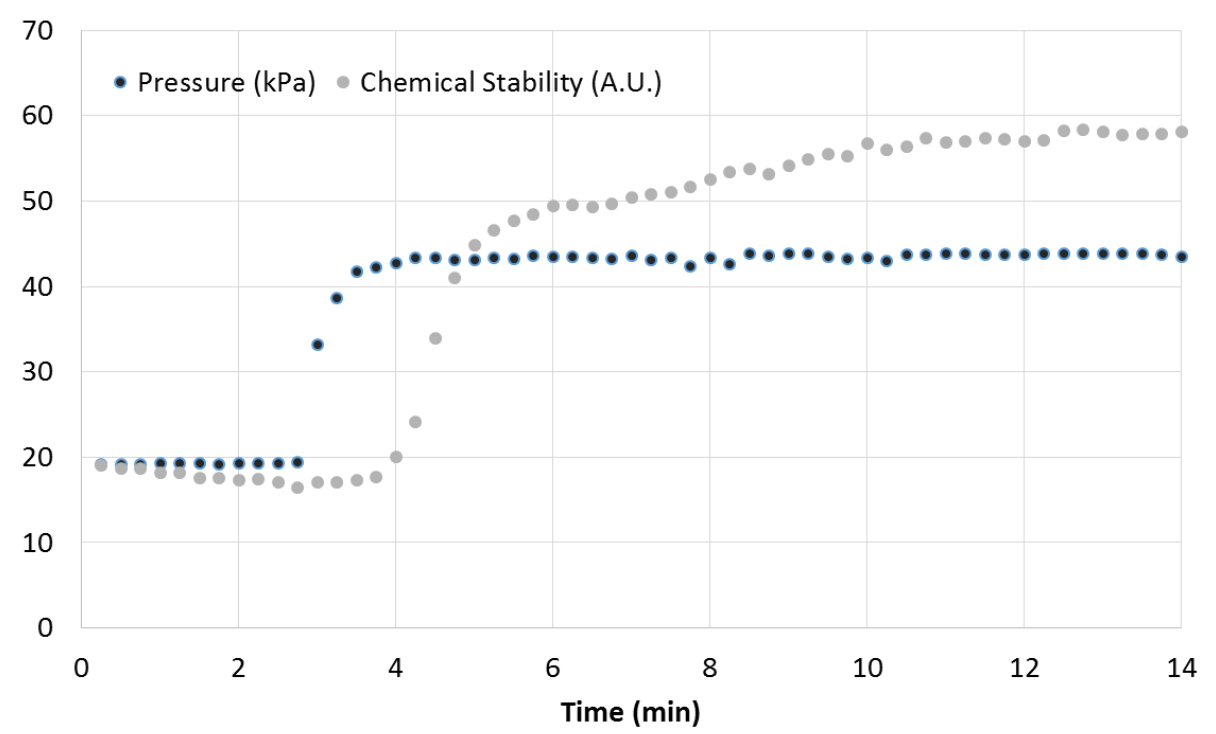

Table 1. Chemical yield in \% product of acetophenone, determined by off-line HPLC.

\begin{tabular}{cccccc}
\hline \multirow{2}{*}{ Flow Rate (mL/min) } & \multicolumn{5}{c}{ Temperature } \\
\cline { 2 - 6 } & $-20{ }^{\circ} \mathrm{C}$ & $-10{ }^{\circ} \mathrm{C}$ & $0{ }^{\circ} \mathrm{C}$ & $10{ }^{\circ} \mathrm{C}$ & $20{ }^{\circ} \mathrm{C}$ \\
\hline 2 & 34 & 17 & 15 & 4.5 & 1 \\
4 & 73 & 58 & 31.5 & 7.5 & 2.28 \\
6 & 80 & 68.5 & 32.5 & 8.5 & 3 \\
8 & 81 & 73 & 37 & 14 & 5 \\
10 & 81 & 74 & 37 & 14.5 & 5 \\
\hline
\end{tabular}

The information from physical and chemical sensors allowed for rapid exploration of a design of experiments in the CFR in less than four hours. Using HPLC to validate chemical steady state would have required at least $30 \mathrm{~h}$ of experiment time. These results demonstrate that the real-time monitoring of physical and chemical steady state in a CFR offers significant advantages for optimizing a reactor system when compared with systems that employ either physical or chemical techniques. This was accomplished through the construction of a reactor designed for PAT implementation using NeSSI sampling systems and Raman spectroscopy. The fast optimization technique is chemistry and hardware agnostic, making this approach a viable means of optimizing any CFR for any suitable chemical reaction.

\section{Conclusions}

On-line analytics, providing information on physical stability combined with Raman spectroscopy and multivariate modeling, allowed for real-time detection of steady state in a CFR. The investigation of a design space to optimize reactor conditions was improved through the rapid detection of steady 
state. The design of experiments was explored in under four hours, with improved confidence in the reproducibility of quality product. In addition, the optimization was achieved via a chemistry agnostic platform that allows for determination of optimum parameters for future production based on quality, volume, and energy needs, regardless of chemistry. Application of advanced chemometrics methods coupled with HPLC information enables a quantitative real-time approach to chemical conversion and could have a broad impact on many process development, optimization, and control methodologies.

\section{Acknowledgments}

The authors of this paper would like to acknowledge William Cost at Parker Hannefin and Brian Phillips at University of Washington for their contributions. This project was supported in part by an appointment to the Research Participation Program at the Center for Drug Evaluation and Research administered by the Oak Ridge Institute for Science and Education through an interagency agreement between the U.S. Department of Energy and the U.S. Food and Drug Administration.

\section{Conflicts of Interest}

The authors declare no conflict of interest.

\section{References}

1. Wiles, C.; Ngamsom, B. Continuous Flow Esterifications; Application Note: chemtrix.com, 2010.

2. Roberge, D.M.; Ducry, L.; Bieler, N.; Cretton, P.; Zimmermann, B. Microreactor technology: A revolution for the fine chemical and pharmaceutical industries? Chem. Eng. Technol. 2005, 28, 318-323.

3. Roberge, D.M.; Bieler, N.; Mathier, M.; Eyholzer, M.; Zimmermann, B.; Barthe, P.; Guermeur, C.; Lobet, O.; Moreno, M.; Woehl, P. Development of an industrial multi-injection microreactor for fast and exothermic reactions-Part II. Chem. Eng. Technol. 2008, 31, 1155-1161.

4. Roberge, D.M.; Zimmermann, B.; Rainone, F.; Gottsponer, M.; Eyholzer, M.; Kockmann, N. Microreactor technology and continuous processes in the fine chemical and pharmaceutical industry: Is the revolution underway? Org. Process Res. Dev. 2008, 12, 905-910.

5. Watts, P.; Wiles, C. Recent advances in synthetic micro reaction technology. Chem. Commun. 2007, doi:10.1039/B609428G.

6. Roberto, M.F.; Dearing, T.I.; Martin, S.; Marquardt, B.J. Integration of continuous flow reactors and online raman spectroscopy for process optimization. J. Pharm. Innov. 2012, 7, 69-75.

7. Wiles, C.; Watts, P.; Haswell, S.J.; Pombo-Villar, E. The aldol reaction of silyl enol ethers within a micro reactor. Lab Chip 2001, 1, 100-101.

8. Wiles, C.; Watts, P.; Haswell, S.J.; Pombo-Villar, E. 1,4-addition of enolates to $\alpha, \beta$-unsaturated ketones within a micro reactor. Lab Chip 2002, 2, 62-64.

9. Leung, S.A.; Winkle, R.F.; Wootton, R.C.R.; deMello, A.J. A method for rapid reaction optimisation in continuous-flow microfluidic reactors using online raman spectroscopic detection. Analyst 2005, 130, 46-51. 
10. Pawliszyn, J. Comprehensive Sampling and Sample Preparation: Analytical Techniques for Scientists; Elsevier: Amsterdam, The Netherlands, 2012; Volume 1.34.

11. Undey, K.; Low, D.; Menezes, J.; Koch, M. Pat Applied in Biopharmaceutical Process Development and Manufacturing: An Enabling Tool for Quality-by-Design; CRC Press: Boca Raton, FL, USA, 2012.

12. Kawaguchi, T.; Miyata, H.; Ataka, K.; Mae, K.; Yoshida, J. Room-temperature swern oxidations by using a microscale flow system. Angew. Chem. Int. Ed. 2005, 44, 2413-2416.

13. Van der Linden, J.J.M.; Hilberink, P.W.; Kronenburg, C.M.P.; Kemperman, G.J. Investigation of the moffatt-swern oxidation in a continuous flow microreactor system. Org. Process Res. Dev. 2008, 12, 911-920.

14. Mozharov, S.; Nordon, A.; Littlejohn, D.; Wiles, C.; Watts, P.; Dallin, P.; Girkin, J.M. Improved method for kinetic studies in microreactors using flow manipulation and noninvasive raman spectrometry. J. Am. Chem. Soc. 2011, 133, 3601-3608.

15. Cao, E.; Sankar, M.; Firth, S.; Lam, K.F.; Bethell, D.; Knight, D.K.; Hutchings, G.J.; McMillan, P.F.; Gavriilidis, A. Reaction and raman spectroscopic studies of alcohol oxidation on gold-palladium catalysts in microstructured reactors. Chem. Eng. J. 2011, 167, 734-743.

16. Mechtilde, S.; Eduard, S.; Andreas, F. Computer controlled chemical micro-reactor. J. Phys. Conf. Ser. 2006, 1, 115.

17. Misra, M.; Yue, H.H.; Qin, S.J.; Ling, C. Multivariate process monitoring and fault diagnosis by multi-scale pca. Comput. Chem. Eng. 2002, 26, 1281-1293.

18. Chen, J.; Liu, K.-C. On-line batch process monitoring using dynamic PCA and dynamic PLS models. Chem. Eng. Sci. 2002, 57, 63-75.

19. Dearing, T.I.; Thompson, W.J.; Rechsteiner, C.E., Jr.; Marquardt, B.J. Characterization of crude oil products using data fusion of process raman, infrared, and nuclear magnetic resonance (NMR) spectra. Appl. Spectrosc. 2011, 65, 181-186.

20. Szostak, R.; Mazurek, S. Quantitative determination of acetylsalicylic acid and acetaminophen in tablets by FT-Raman spectroscopy. Analyst 2002, 127, 144-148.

21. Shah, R.B.; Tawakkul, M.A.; Khan, M.A. Process analytical technology: Chemometric analysis of raman and near infra-red spectroscopic data for predicting physical properties of extended release matrix tablets. J. Pharm. Sci. 2007, 96, 1356-1365.

(C) 2013 by the authors; licensee MDPI, Basel, Switzerland. This article is an open access article distributed under the terms and conditions of the Creative Commons Attribution license (http://creativecommons.org/licenses/by/3.0/). 